\title{
Supplementary Materials \\ Long-range charge reorganization as an allosteric control signal in proteins
}

Koyel Banerjee-Ghosh,\# Shirsendu Ghosh,\# Hisham Mazal, Inbal Riven, Gilad Haran,* Ron Naaman,*

\section{Supplementary Text}

\section{Chiral-induced spin selectivity and proteins}

The spin is the intrinsic angular momentum of the electron and can have two values, frequently referred to as spin UP or spin DOWN. The common electron pair bond is often considered to possess one electron of the UP spin and another of the DOWN spin. In the last decade, it has been established that the efficiency of conduction of electrons through chiral molecules depends on their spin. Which spin is preferred depends on the handedness of the molecule, and is governed by the CISS effect. ${ }^{1}$ A ferromagnetic substrate that is magnetized perpendicular to its surface, has most of its electrons pointing either UP or DOWN depending on the direction of magnetization. Hence, if a molecule that is bound to a ferromagnetic substrate is chiral, the ability to inject efficiently electrons into the molecules or from the molecules into the substrate depends on the spin of the electrons, namely on the direction of magnetization of the ferromagnet. Hence, a ferromagnet and a chiral molecule attached to it serve as a "valve" that controls the charge flow (See Fig. 1). In our experiment the chiral molecule can be the protein itself or a chiral linker, as shown below. While earlier studies have shown that the CISS effect can be manifested in proteins, 2,3 in this work we used this effect to control charge migration into a surface-bound antibody by changing the spin direction of electrons injected into the protein without altering the binding of the antibody to the metal substrate.

\section{Control experiments}

The CISS effect and the anti-His antibody. To prove that the CISS effect is operative with the anti-His antibody used in this study, we adsorbed the protein on a Hall device (see Supplementary Method and Fig. S1A for details). Upon applying an external electric field, we observed a Hall voltage that indicated spin-selective charge flow. Figures S1B and C 
present the Hall signal measured for various gate voltages applied on the adsorbed antibody molecules. Details on the experimental setup for the Hall measurement can be found in Ref. 4. Indeed, the results indicated that applying an electric field on the antibody generates a spin-dependent charge reorganization.

Measuring the background when the anti-His antibody is immobilized through D- or L-cysteine on gold surface. Two control experiments were carried out to check background signals. In the first experiment, L-cysteine coated samples were exposed to the antigen solution for $2 \mathrm{~s}$ and $8 \mathrm{~s}$ without the antibody. The average number of $\mathrm{ClpB}$ molecules attached on L-cysteine coated surfaces were found to be $0.3 \pm 0.15$ for $2 \mathrm{~s}$ and $1.83 \pm 0.4$ for $8 \mathrm{~s}$. In another experiment, the L-cysteine carboxyl group esterification step was skipped and the surfaces were incubated within the antibody solution for $30 \mathrm{mins}$ at room temperature and exposed to the antigen solution for $2 \mathrm{~s}$ and $8 \mathrm{~s}$. In this case, the average number of $\mathrm{ClpB}$ attached was $2.34+0.43$ for $2 \mathrm{~s}$ and $2.31+0.41$ for $8 \mathrm{~s}$. Hence, the number of $\mathrm{ClpB}$ molecules adsorbed on the surface and detected in these two control experiments was not significant compared to the experiment with fully coupled antibody molecules.

\section{Supplementary Methods}

\section{Polarization Modulation Infrared Reflectance Absorption Spectroscopy (PMIRRAS)}

PMIRRAS was used to characterize the cysteine and oligopeptide monolayers on gold surface, which were dried after their formation. Each spectrum was obtained by accumulating 1000 scans with the samples mounted at Brewster angle. The spectra are shown in Figure S6.

The spectra of the L- and D-cysteine monolayers (Figure S6A) show characteristic peaks at $\sim 3250 \mathrm{~cm}^{-1}$ (stretching mode of the $\mathrm{OH}$ of carboxylic group), $\sim 1640 \mathrm{~cm}^{-1}$ (bending mode of $\mathrm{NH}$ ), $\sim 1460 \mathrm{~cm}^{-1}$ (bending mode of $\mathrm{CH}$ ) and $\sim 1140 \mathrm{~cm}^{-1}$ ((stretching mode of $\mathrm{CN}$ ). There is also a signature of $\mathrm{CH}$ stretching mode at $\sim 2850 \mathrm{~cm}^{-1}$ and $\sim 2920$ $\mathrm{cm}^{-1}$. As the intensity of peaks for both cysteine enantiomers are almost same, similar coverage of the surface is inferred.

The spectra of the oligopeptide monolayers (Figure S6B) show two characteristic peaks at $\sim 1670$ and $\sim 1540 \mathrm{~cm}^{-1}$, typical of amide-I (stretching mode of the CO bond) and 
amide-II ( $\mathrm{N}-\mathrm{H}$ in- plane bending mode and $\mathrm{C}-\mathrm{N}$ stretching mode), respectively. The similar intensity of the peaks of L- and D- oligopeptide proves similar coverage of the surface.

Hall voltage measurement. We measured Hall voltage generated by the Ultra-LEAFTM Purified anti-His antibody (purchased from BioLegend, catalog number \# 652508) monolayer. To this end, we generated a self-assembled monolayer (SAM) of the anti His tag antibody on top of a $5 \mathrm{~nm}$ gold-coated Hall device. The fabrication of Hall devices is reported in Ref. 5. In brief, an $\mathrm{AlGaN} / \mathrm{GaN}$ wafer on a sapphire substrate (from NTT) was used. The wafer was composed of a nucleation layer, and then an intrinsic GaN layer of $180 \mathrm{~nm}$. On top of the i-GaN layer, an intrinsic-AlGaN layer with $20 \mathrm{~nm}$ thickness was present. Finally, a capping layer of GaN with $2 \mathrm{~nm}$ thickness was present on top of the i$\mathrm{AlGaN}$ layer. Using this wafer, Hall devices were fabricated by standard photolithography in a class 1000 clean room. To prepare ohmic contacts, a metallic multilayer [Ti $(20 \mathrm{~nm}) / \mathrm{Al}$ $(100 \mathrm{~nm}) / \mathrm{Ni}(40 \mathrm{~nm}) / \mathrm{Au}(40 \mathrm{~nm})]$ was annealed at $850^{\circ} \mathrm{C}$. The channel, $500 \mu \mathrm{m}$ in length and $40 \mu \mathrm{m}$ in width, was coated with $5 \mathrm{~nm}$ Au for molecular adsorption. The device is schematically presented in the Figure S1A. Hall devices were cleaned for measurement by boiling in acetone and ethanol. Then the devices were glued to a sample holder and connected using wire bonding. All the connections were passivated to avoid shortcircuiting. For measurements, a $200 \mu \mathrm{L}$ cell made up of PDMS (polydimethylsiloxane) was glued onto the device holder. Then a monolayer of anti-His Tag antibody was attached to gold-coated surface of the device using dithiobis[succinimidyl]propionate (DSP, purchased from Sigma Aldrich, purity $>97 \%$ ) as a linker. A DSP monolayer was formed on the gold surface by dropcasting the solution of DSP in DMSO $(4 \mathrm{mg} / \mathrm{ml})$ on the device and kept for 30 mins. After rinsing the surfaces with DMSO and water, antibody solution in PBS $(1 \mathrm{mg} / \mathrm{ml})$ was drop-casted on the device and incubated for $4 \mathrm{~h}$. The device was rinsed with PBS and filled with $200 \mu$ of PBS. For polarization experiments, one side of a glass coverslip coated with gold was used as gate electrode to provide the electric field for the polarization of the molecules. The whole measurement was done in a dark Faraday cage. A constant current of $10 \mu \mathrm{A}$ was applied between the source (S) and the drain (D) electrodes using a Keithley 2636B source meter. Gate voltage was applied using another 
power source Keithley 2636A. A 2182A Nanovoltmeter was connected between two Hall probes to measure the Hall voltage. 


\section{Supplementary Figures}

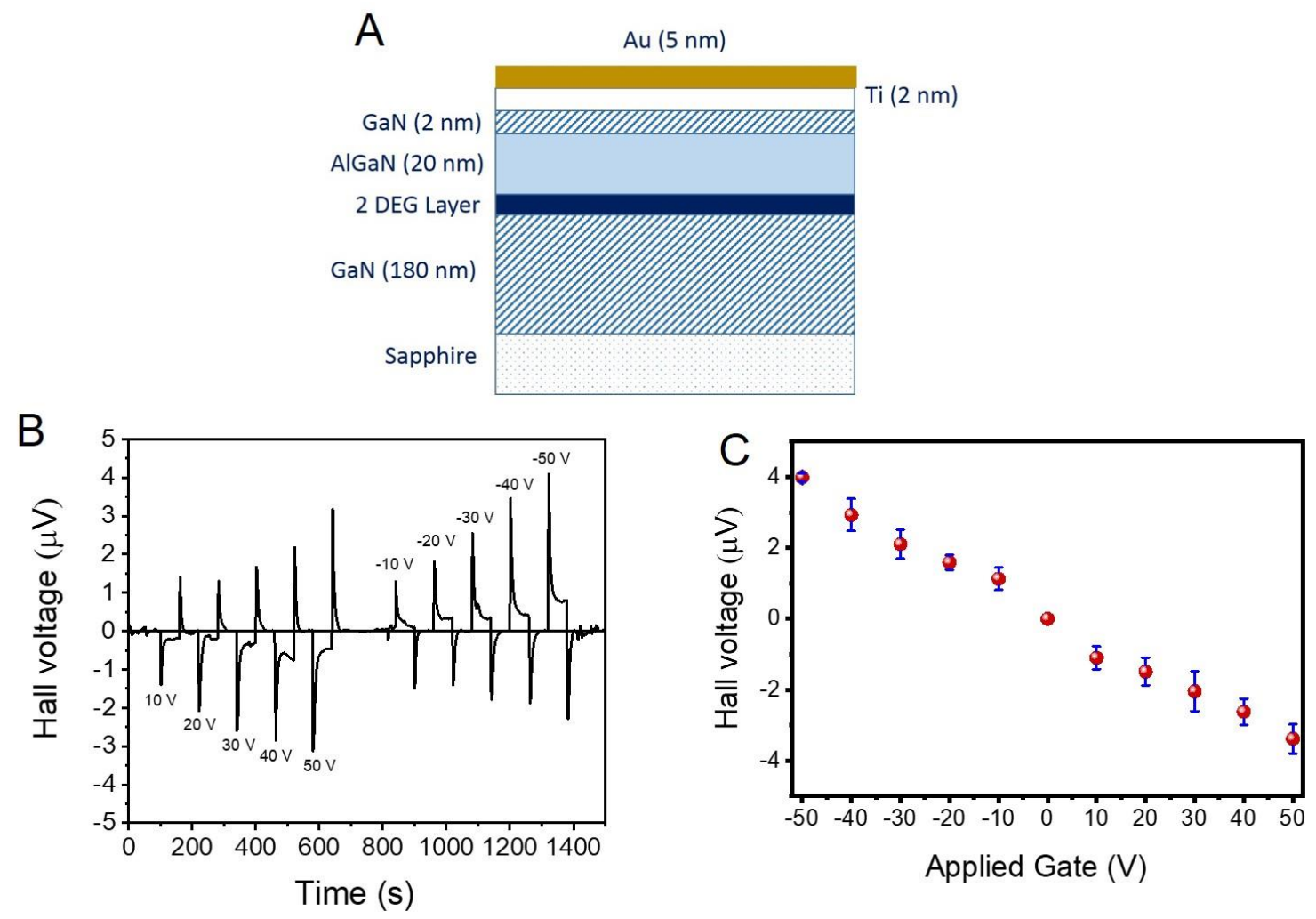

Fig. S1. Hall voltage measurement of anti-His tag antibodies polarized by an electric field. A. Schematic presentation of the Hall device. Antibodies were immobilized on the gold surface. B. Gate-voltage dependent Hall voltage measurement conducted on a device coated with anti-His tag antibodies. Upon applying an external electric field, the antibody is charge-polarized and a Hall voltage is observed, which indicates that the charge flow is spin selective. C. Dependence of Hall voltage on applied gate voltage. With an increase in applied gate voltage, Hall voltage is enhanced. 


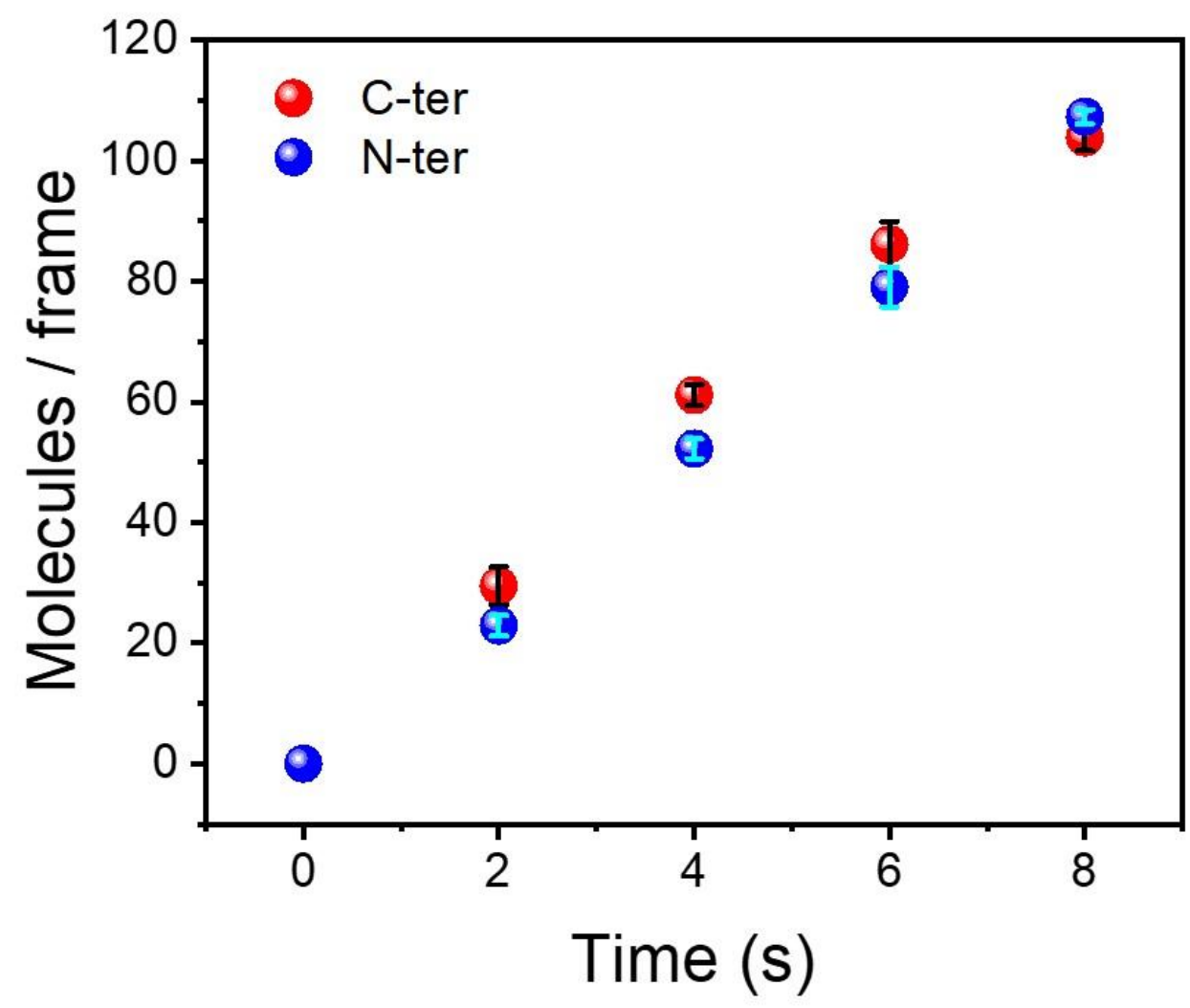

Fig. S2. No difference in association rate in the absence of the magnetic field. Reaction kinetics of the antibody molecules with C-terminal His-tagged ClpB and N-terminal Histagged $\mathrm{ClpB}$ in the absence of a magnetic field. The antibody is immobilized on a $2 \mathrm{~nm}$ gold coated ferromagnetic surface. 
A

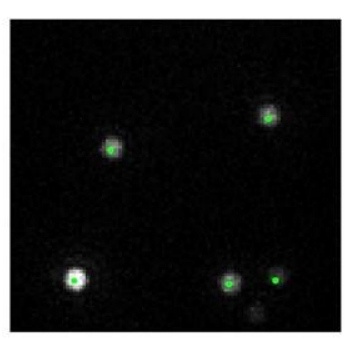

C

Number of Change Point $=2$

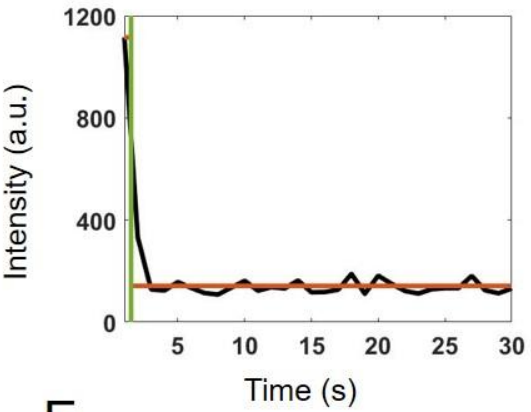

$E$ Number of Change Point $=2$

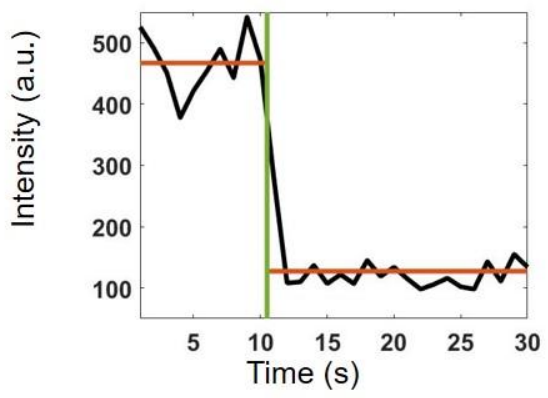

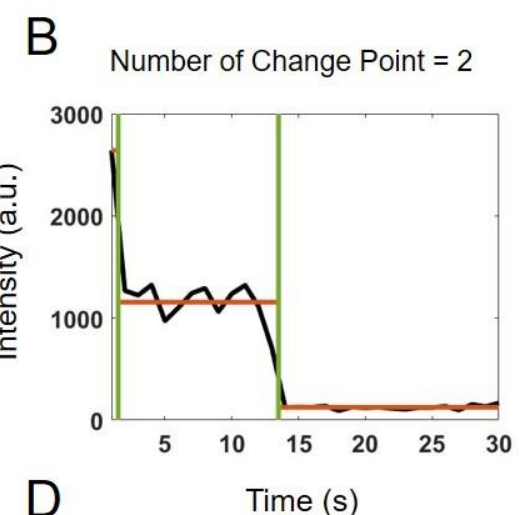

Number of Change Point $=2$

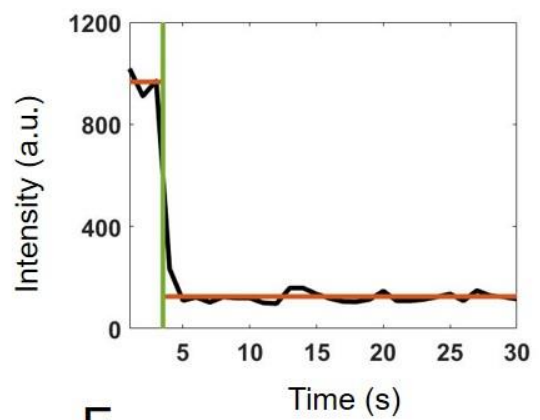

$\mathrm{F}$

Number of Change Point $=2$

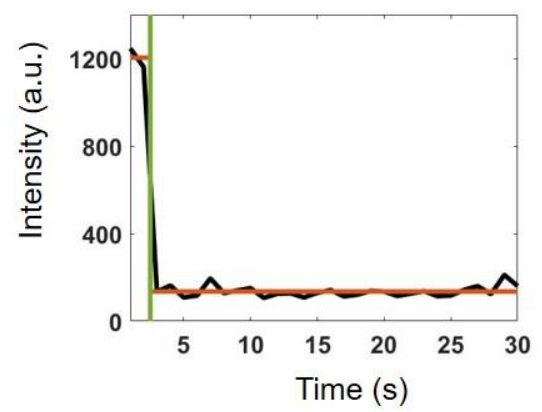

Fig. S3. Counting molecules. A. Representative image of ClpB molecules attached on a gold surface. Green dots indicate the detected locations of centers of mass of individual emitters. (B-F) Intensity verses time trajectories of individual emitters from (A). Green lines shows the change points in the intensity verses time traces. Orange line indicates the average intensity in the specified regions. 


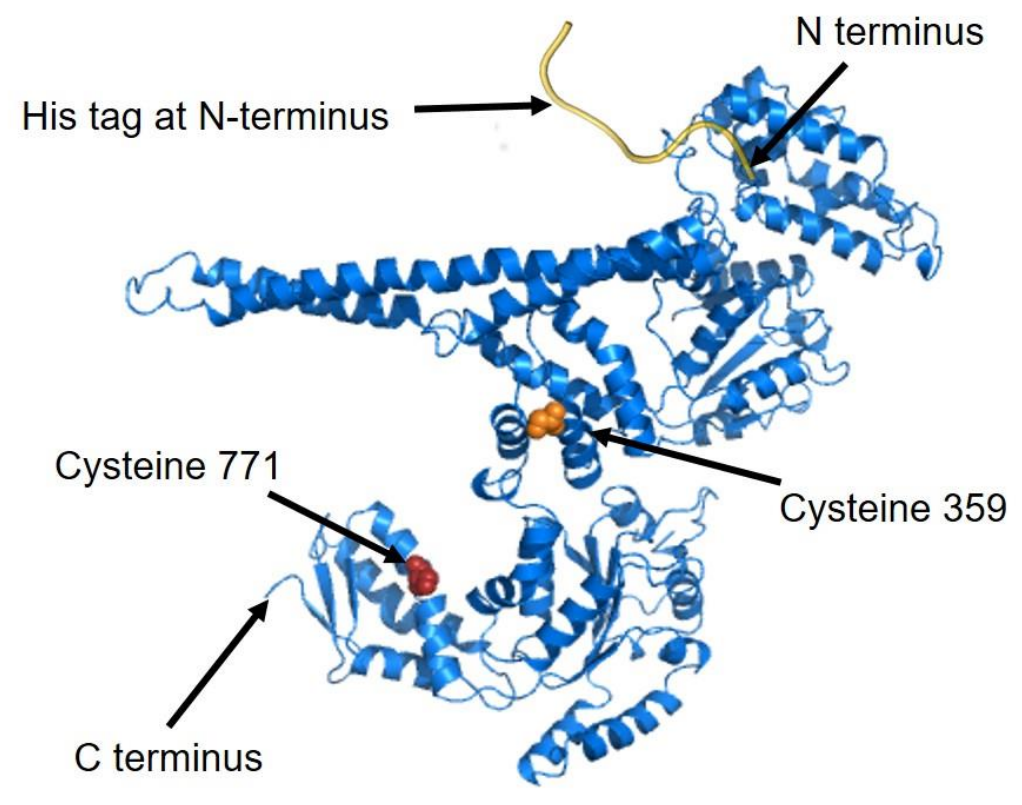

Fig. S4. Monomeric structure of the protein ClpB used in the experiments, shown as a cartoon (PDB: 1QVR). His-tag at the N-terminus is shown in yellow, orange and red spheres indicate the position of the free cysteine mutations, S359C and S771C. The fluorescent dye labels either of these cysteines. 

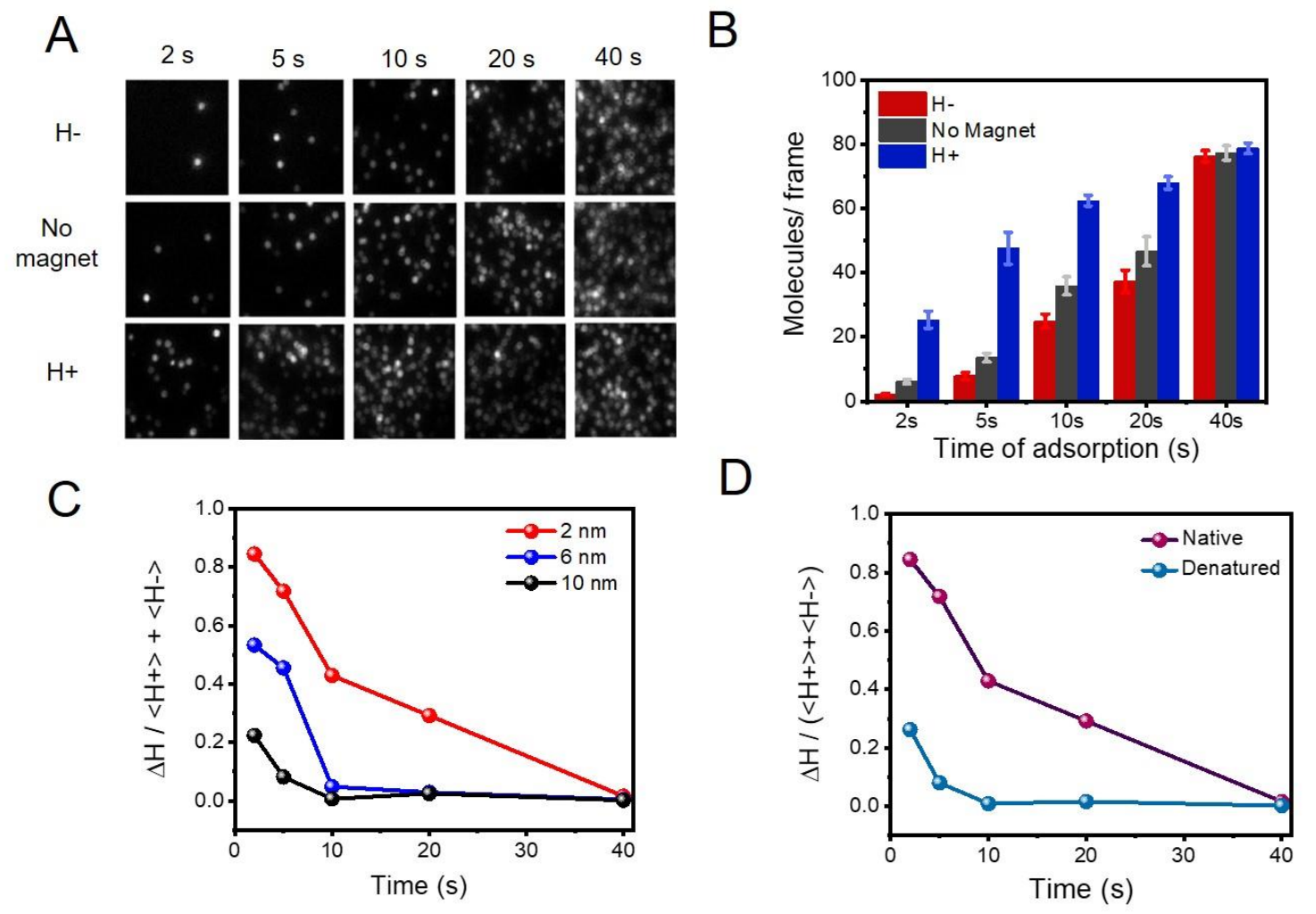

Fig. S5. Adsorption kinetics of native and denatured ClpB on a gold coated magnetic surface. A. Adsorption kinetics on a $2 \mathrm{~nm}$ Au coated magnetic surface. Microscope images of individual fluorescent ClpB molecules adsorbed at different time intervals: $2 \mathrm{sec}, 5 \mathrm{sec}, 10 \mathrm{sec}, 20 \mathrm{sec}$ and 40 sec, with two different magnet orientations. B. Histogram of the number of adsorbed protein molecules as a function of time for different magnet orientations. The number of molecules was calculated as described in the Methods section. C. Spin selectivity of the rate of protein adsorption with gold layers of different thickness: $2 \mathrm{~nm}, 6 \mathrm{~nm}$ and $10 \mathrm{~nm} ; \Delta \mathrm{H}=<\mathrm{H}+>-<\mathrm{H}->$. D. Spin selectivity of adsorption rates of native and denatured $\mathrm{ClpB}$ molecules on a $2 \mathrm{~nm}$ Au-coated magnetic surface 

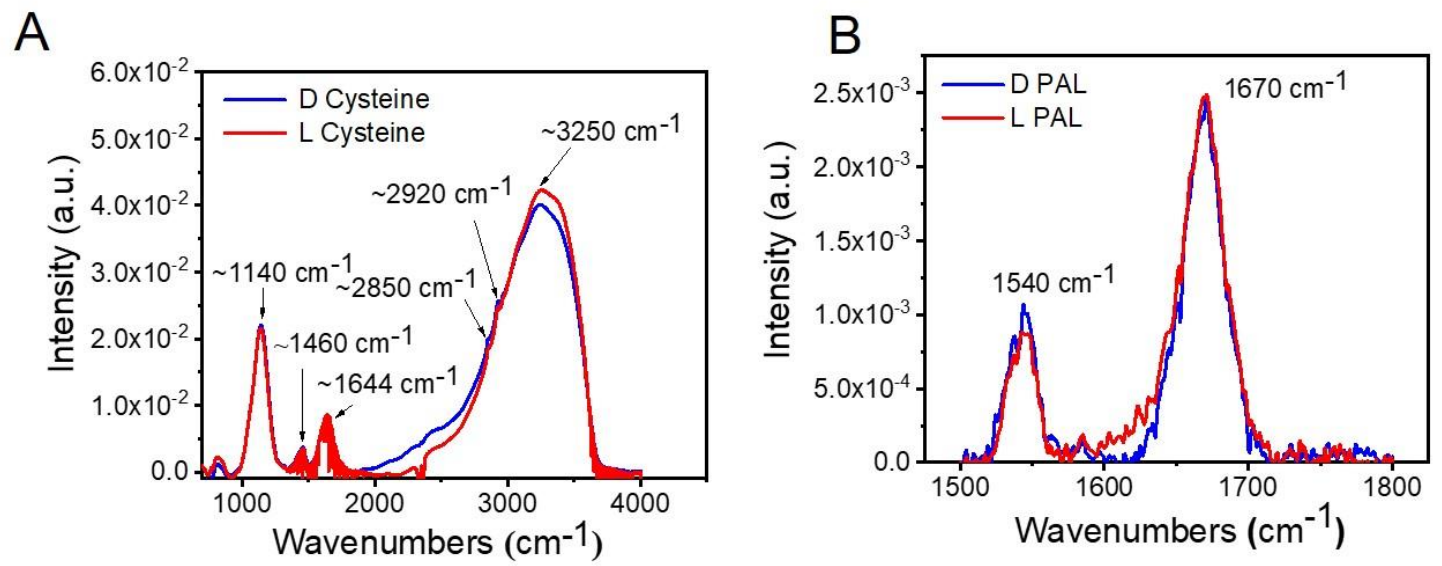

Fig. S6. Polarization Modulation Infrared Reflectance Absorption Spectroscopy of various monolayers used in the experiments. A. D- and L- cysteine. B. D-and L PAL.

1. Naaman, R.; Waldeck, D. H. Spintronics and Chirality: Spin Selectivity in Electron Transport Through Chiral Molecules. Ann. Rev. Phys. Chem. 2015, 66, 263-81.

2. Mondal, P.; Fontanesi, C.; Waldeck, D. H.; Naaman, R. Spin-dependent Transport through Chiral Molecules Studied by Spin-dependent Electrochemistry. Acc. Chem. Res. 2016, 49, 2560-2568.

3. Mishra, S.; Pirbadian, S.; Mondal, A. K.; El-Naggar, M. Y.; Naaman, R. SpinDependent Electron Transport through Bacterial Cell Surface Multiheme Electron Conduits. J. Am. Chem. Soc. 2019, 141, 19198-19202.

4. Fontanesi, C.; Capua, E.; Paltiel, Y.; Waldeck, D. H.; Naaman, R. Spin Dependent Processes Measured without a Permanent Magnet. Adv. Mat. 2018, 30, 1707390.

5. Mishra, S.; Poonia, V. S.; Fontanesi, C.; Naaman, R.; Fleming, A. M.; Burrows, C. J. Effect of Oxidative Damage on Charge and Spin Transport in DNA. J. Am. Chem. Soc. 2019, 141 (1), 123-126. 